\title{
Cineforum-virtual criminológico como estrategía de aprendizaje teorico-práctico para el análisis del comportamiento criminal en el Grado de Criminología
}

\author{
Lucía Aparicio Chofréa , Carla de Paredes Gallardo ${ }^{b}$ y Cristina Escamilla Robla ${ }^{c}$ \\ a (Universidad Europea de Valencia y Universidad de Valencia, \\ lucia.aparicio@universidadeuropea.es), b(Universidad Europea de Valencia, \\ carla.deparedes@universidadeuropea.es) y c(Universidad Europea de Valencia, \\ mariacristina.escamilla@universidadeuropea.es).
}

\begin{abstract}
It deals with a cinematographic activity in the classroom and in a virtual context, with consecutive debates, in order to analyze behaviors and to elaborate, where appropriate, the reports or projects, main competences of the studies of the Degree of Criminology. This activity has been integrated in the 3rd academic year 2015-2016, with a multidisciplinary, progressive and integrative character. It is part of the practical work of the subjects, which in turn are integrated with each other. Due to the difficulty of being able to present students with real cases of crime, the films were carefully selected in order to be able to observe many of the actions, factors and indications that concur in these events. In this way, the student was able to analyze and evaluate, as demanded on the events developed, to later elaborate his practical work. The films were projected during the last month of each quarter, so that the students had knowledge of the case to be treated. They were also available on the virtual platform of the University so they can review those difficult scenes to interpret or the signs to assess. The results on the evaluation of this resource conclude that the students have worked with a high level of motivation, a greater internalization of knowledge and a more applied and creative learning.
\end{abstract}

Keywords: Analysis of film viewing, practical and virtual application, integration, motivation.

\footnotetext{
Resumen

Trata de una actividad cinematográfica en aula y en contexto virtual, con los debates consecutivos, con el fin de analizar conductas y elaborar, en su caso, los informes o proyectos, competencias principales de los estudios del Grado de Criminología. Esta actividad se ha integrado en el $3^{\text {er }}$ curso académico 2015-2016, con carácter multidisciplinar, progresivo e integrador. Forma parte de los trabajos prácticos de las asignaturas, que a su vez se integran unas con otras. Debido a la dificultad de poder presentar al alumnado casos reales sobre criminalidad, se seleccionaron las películas cuidadosamente con el fin de poder observar muchas de las acciones, factores e indicios que
} 
concurren en dichos sucesos. De esta manera, el alumno pudo analizar y evaluar, según lo demandado sobre los acontecimientos desarrollados, para posteriormente elaborar su trabajo práctico. Las películas se proyectaron durante el último més de cada trimestre, para que los alumnos tuvieran conocimientos del caso a tratar. También estuvieron disponibles en la plataforma virtual de la Universidad para que puedan volver a visionar aquellas escenas más díficiles de interpretar o los indicios a valorar. Los resultados sobre la valoración de este recurso concluyen que los estudiantes han trabajado con un alto nivel de motivación, una mayor interiorización de los conocimientos y un aprendizaje más aplicado y creativo.

Palabras clave: Análisis de visionado de películas, aplicación práctica y virtual, integración, motivación.

\section{Introducción}

El cine, como recreación humana, es de las más extraordinarias que existen desde la perspectiva de la comunicación. Toda la información que codifica el individuo que visiona una película, describe situaciones reales o fictícias y genera emociones, cuyo análisis posterior es útil para valorar diferentes hechos, conductas e incluso para provocar actitudes y acciones distintas a las habituales (Icart-Isern, 2008). Desde la enseñanza primaria y secundaria, se ha ido utilizando el cine comercial con fines formativos (Martínez, 2003), incluso en educación superior, en áreas específicas, como por ejemplo, medicina (GarcíaSánchez, Fresnadillo y García-Sánchez, 2002), o en farmacología (Bosch y Baños, 1999).

Los avances técnológicos que popularizaron el séptimo arte, surgieron en la última década del siglo XX, donde apareció el "home cinema", así como el DVD, la televisión digital, entre otros. Esta evolución permite conseguir con facilidad dichos materiales, incluso para uso exclusivo de aquel que quisiera disfrutarlo para sí mismo. De esta manera, el cine se convierte en un medio de comunicación de masas (De la Torre, 1996).

El lenguaje cinematográfico trae consigo diferentes niveles de comunicación. Por una parte, se encuentra el nivel de los sistemas perceptivos, como la vista y el oído, combinándose con lenguajes asociados, como la música y los efectos especiales; además, actúa un tercer nivel donde se pone en marcha procesos subconscientes, como los encuadres, los espacios escénicos, los movimientos, etc., (Domínguez y Guercia, 2005), que juntos con los anteriores hace de este recurso, en un contexto educativo, uno de los más óptimos para la adquisición de conocimientos, habilidades y actitudes, cuando se acompaña por docentes que comparten los códigos de este lenguaje (Icart-Isern, 2008).

El cine formativo utilizado frecuentemente, según de la Torre, catedrático de Didáctica e Innovación Educativa (Universitat de Barcelona), es "la emisión y recepción intencional de películas portadoras de valores culturales, humanos, técnico-científicos o artísticos, con la finalidad de mejorar el conocimiento, las estrategias o las actitudes y opiniones de los espectadores". Asimismo, enfocando el cine con un carácter formativo, desde su argumento y con la intención de promover la reflexión y el análisis de un tema, problema, situación o

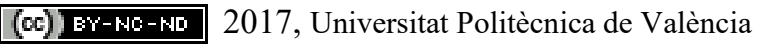


acontecimientos, se convierte en un proceso dínamico, creativo y de complicidad entre el docente y el alumno (Icart-Isern, 2008).

Las nuevas tecnologías son un valor añadido a la transmisión de conocimientos y un cambio del modelo docente en los procesos de enseñanza-aprendizaje para el desarrollo de competencias, y donde el docente es el encargado de guiar, planificar y evaluar el método utilizado. Un buen ejemplo de la utilización del cine dentro de la metodología docente, es el desarrollado por Pérez y López (2007) en sus enseñanzas de recursos humanos:

\begin{abstract}
A través del cine el alumno/espectador puede recibir información que le ayude a ordenar cognitivamente sus conocimientos, dándole la oportunidad de participar en muchas situaciones diferentes que, de no ser por este medio, no hubiera tenido a su alcance. El cine propone al espectador una historia, facilitando la identificación del espectador con los diferentes matices del discurso. A través de la identificación de los personajes, la trama, la acción, el espectador pasa de ser mero testigo de la narración a convertirse en actor esta. De esta forma el aprendizaje se produce activamente, a través de la experiencia (Pérez y López, 2007:66).
\end{abstract}

Otro ejemplo sobre la utilización del cine en las enseñanzas, en este caso en Sociología, confirman los profesores Fernández y Olmedo (2003) tras su experiencia sobre el apoyo audiovisual. Describen que puede ser un camino eficaz para presentar de manera comprensible la materia y conseguir que atraiga y despierte el interés de los alumnos hacia ella y les resulte relevante.

Trabajos como el de Betanzo (2010), obtuvieron valores positivos, destacando la conveniencia de abordar los conceptos con carácter multidisciplinar en lugar de abordarlos de forma aislada, así como la importancia de la motivación en los procesos de aprendizaje con la inclusión de medios audiovisuales.

Este proyecto se integró en las enseñanzas del 3er curso de Criminología y durante todo el curso académico 2015-2016, proyectando las películas a medida que se iban adquiriendo los conocimientos adecuados de cada asignatura. Posteriormente, con un guión previo, se trabajaron los casos, conceptos, situaciones, etc., pertinentes relacionadas con los contenidos a tratar. Al finalizar el curso, se valoró este proyecto con mucha satisfacción, tanto por parte de los alumnos como del profesorado afectado.

Este recurso, con una selección adecuada de películas, ofrece una visión global y a la vez individual sobre los elementos que estudia la Criminología como el delincuente, el delito y la víctima, además del control social, para la prevención de la delincuencia. Además, ayuda al estudiante a analizar la presencia del fenómeno delictivo en toda su extensión y con las distintas dimensiones de estudio.

En conclusión, el presente trabajo tuvo como fin la utilización de la actividad cinematográfica como innovación docente en la enseñanza universitaria, con carácter multidisciplinar, integrador y progresivo. Multidisciplinar porque una misma película va ser utilizada para diferentes asignaturas; integrador, porque dichas asignaturas están relacionadas en sus contenidos y própositos finales, y otras, en los procesos que se 
desarrollan dentro del argumento (ejemplo: trastorno de conducta de un agresor que a su vez va a ser juzgado por un tribunal. Psicología + Derecho); y progresivo, porque se va a planificar de forma progresiva para que exista una continuidad en los conocimientos teóricos y prácticos de dichas asignaturas. Todo ello, se ha teniendo en cuenta puesto que en el grado de Criminología se desarrollan asignaturas de diferentes áreas de conocimiento como la Psicología, la Sociología y el Derecho.

A continuación se detallan los objetivos de este recurso; su desarrollo en temporalidad, participantes, material utilizado; la metolodogía llevada a cabo, con un cronograma ejemplo, y breve explicación del procedimiento; resultados de la valoración por parte de los alumnos; y unas conclusiones.

\section{Objetivos}

El objetivo general de este trabajo es desarrollar un recurso formativo en aula y en formato virtual, como innovación docente, para conseguir el aprendizaje aplicado multidisciplinar, integrador y progresivo de los conocimientos y competencias de diversas asignaturas interrelacionadas del Grado de Criminología.

Como objetivos específicos:

- Crear una plataforma en red de profesorado universitario con el recurso "Cineforum-virtual Criminológico", como metodología para la enseñanza de un grupo de asignaturas interrelacionadas.

- Motivar a los estudiantes de Criminología a la asistencia, debate y participación en aula y, posteriormente, también en la plataforma virtual.

- Motivar a los estudiantes al trabajo individual-virtual, al tener la posibilidad de visionar de nuevo y con exahustividad las escenas de las películas necesarias para su análisis.

- Comprender casos-problemas desde una perspectiva global e individual, desde los inicios de los acontecimientos y su evolución, en un caso partícular, hasta las posibles alternativas de solución.

- Afianzar los contenidos teóricos de manera creativa y motivadora.

- Participar de forma activa en el análisis y valoración de casos, así como en la elaboración de los informes pertinentes, en su caso.

- Aprender a debatir y defender argumentos desde toda la información que se recoge en las películas.

- Manejar las emociones para la toma de decisiones más adecuadas.

\section{Desarrollo de la innovación}

A continuación se desarrolla el proceso del recurso formativo de innovación docente, "Cineforum-virtual Criminológico". 


\subsection{Temporalidad}

El “Cineforum-virtual Criminológico", se llevó a cabo durante todo el curso académico 2015-2016, en tres momentos, coincidentes con los tres trimestres: T1, T2 y T3. Se proyectaron a medida que se iban adquiriendo los conocimientos adecuados para poder evaluar, valorar y analizar los acontecimientos que iban sucediendo en el desarrollo de la película seleccionada. Aproximadamente, se proyectaron a partir de la mitad de cada trimestre. Posteriormente, el alumno disponía del recurso en la plataforma o se facilitaba por el profesor para su visionado individual y exhaustivo en casa.

\subsection{Participantes}

Asistieron 11 alumnos, los matriculados en el Grado de Criminología, en el $3^{\text {er }}$ curso. Además, según el argumento de la película proyectada, también asistieron los profesores/as de las asignaturas relacionadas con la misma.

\subsection{Películas proyectadas y asignaturas intervinientes en el proyecto}

Como se puede observar en la Tabla 1, se distribuyen, en primer lugar, las películas que se proyectaron para trabajar las asignaturas de $3^{\circ}$ de Criminología, divididas en dos bloques: el bloque I, todas aquellas que se relacionan entre sí, en algún contenido; y el bloque II, donde con dichas películas o capítulos se podrán observar aquellos indicios sobre criminalística.

Tabla 1. Listado de películas y asignaturas donde se proyectan

\begin{tabular}{|c|c|c|c|c|}
\hline & Películas & Asignaturas $3 \cong$ Criminología & Carácter & ECTS \\
\hline \multirow{8}{*}{$\frac{\bar{g}}{\frac{0}{0}}$} & \multirow{8}{*}{$\begin{array}{l}\text { 1. "El Bola" } \\
\text { 2. "Volando Voy" } \\
\text { 3. "El cadáver de Anna Fritz" } \\
\text { 4. "Musarañas" } \\
\text { 5. "Relatos Salvajes" } \\
\text { 6. "Hard Candy" } \\
\text { 7. "American Psycho" } \\
\text { 8. "Tenemos que hablar de Kevin" } \\
\text { 9. "No hay país para viejos" } \\
\text { 10. "Las dos caras de la verdad" } \\
\text { 11. "Mentes Criminales" } \\
\text { 12. "Celda 211" } \\
\text { 13. "El experimento" }\end{array}$} & $\begin{array}{l}\text { Prevención y Tratamiento de la } \\
\text { Delincuencia }\end{array}$ & $\mathrm{OB}$ & 6 \\
\hline & & Delincuencia y Control Social & $\mathrm{OB}$ & 6 \\
\hline & & Conductas Criminales Tipificadas & $\mathrm{OB}$ & 6 \\
\hline & & Procesos y Procedimientos Penales & $\mathrm{OB}$ & 6 \\
\hline & & $\begin{array}{l}\text { Habilidades para la Intervención } \\
\text { Social y Educativa en el } \\
\text { Delincuente }\end{array}$ & $\mathrm{OB}$ & 6 \\
\hline & & Víctimología & $\mathrm{OB}$ & 6 \\
\hline & & Penología y Derecho Penitenciario & $\mathrm{OB}$ & 6 \\
\hline & & Psiquiatría Forense & $\mathrm{OB}$ & 6 \\
\hline \multirow{2}{*}{$\begin{array}{l}= \\
\frac{0}{2} \\
\frac{0}{2}\end{array}$} & \multirow{2}{*}{$\begin{array}{l}\text { 14. Seven } \\
\text { 15. Series "CSI" }\end{array}$} & Taller de Criminalística I & $\mathrm{OB}$ & 6 \\
\hline & & Taller de Criminalística II & $\mathrm{OB}$ & 6 \\
\hline
\end{tabular}

Fuente: Elaboración propia (2016) 
Cineforum-virtual criminológico como estrategía de aprendizaje teorico-práctico para el análisis del comportamiento criminal en el Grado de Criminología

\subsection{Métodología}

3.4.1. Cronograma ejemplo

$\mathbf{T 1}$ (trimestre 1)

Asig: Delincuencia y Control Social

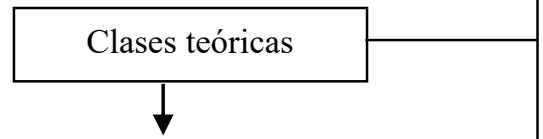

Enlace en T3 con asig:

Habilidades para la Intervención

Social del Delincuente

\section{T1 (trimestre 1)}

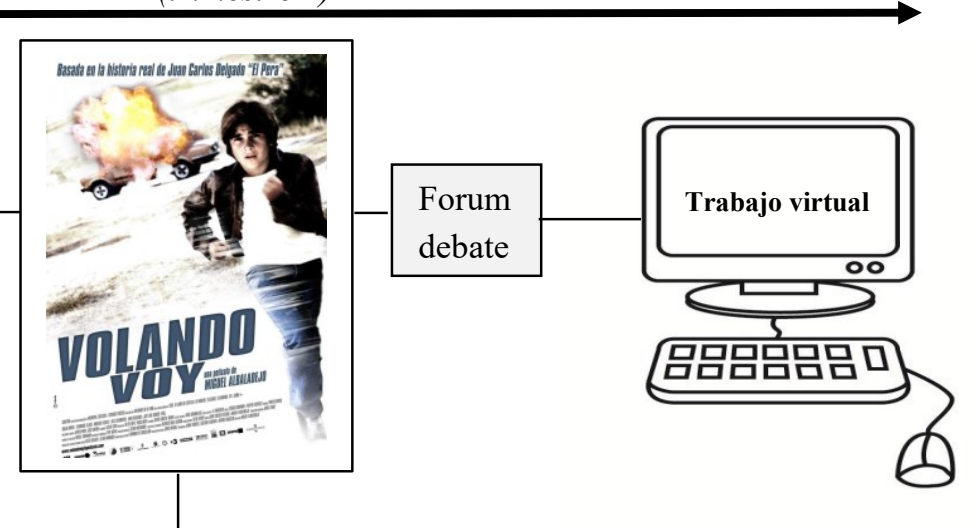

Asig: Conductas Criminales Tipificadas

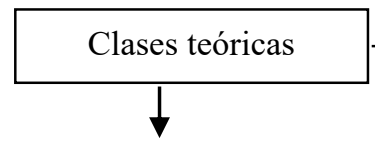

Enlace en T3 con asig:

Psiquiatría Forense

Asig: Procesos y Procesimientos Penales

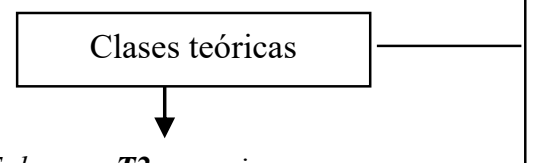

Enlace en $\boldsymbol{T} 2$ con asig:

Penología y Derecho Penitenciario

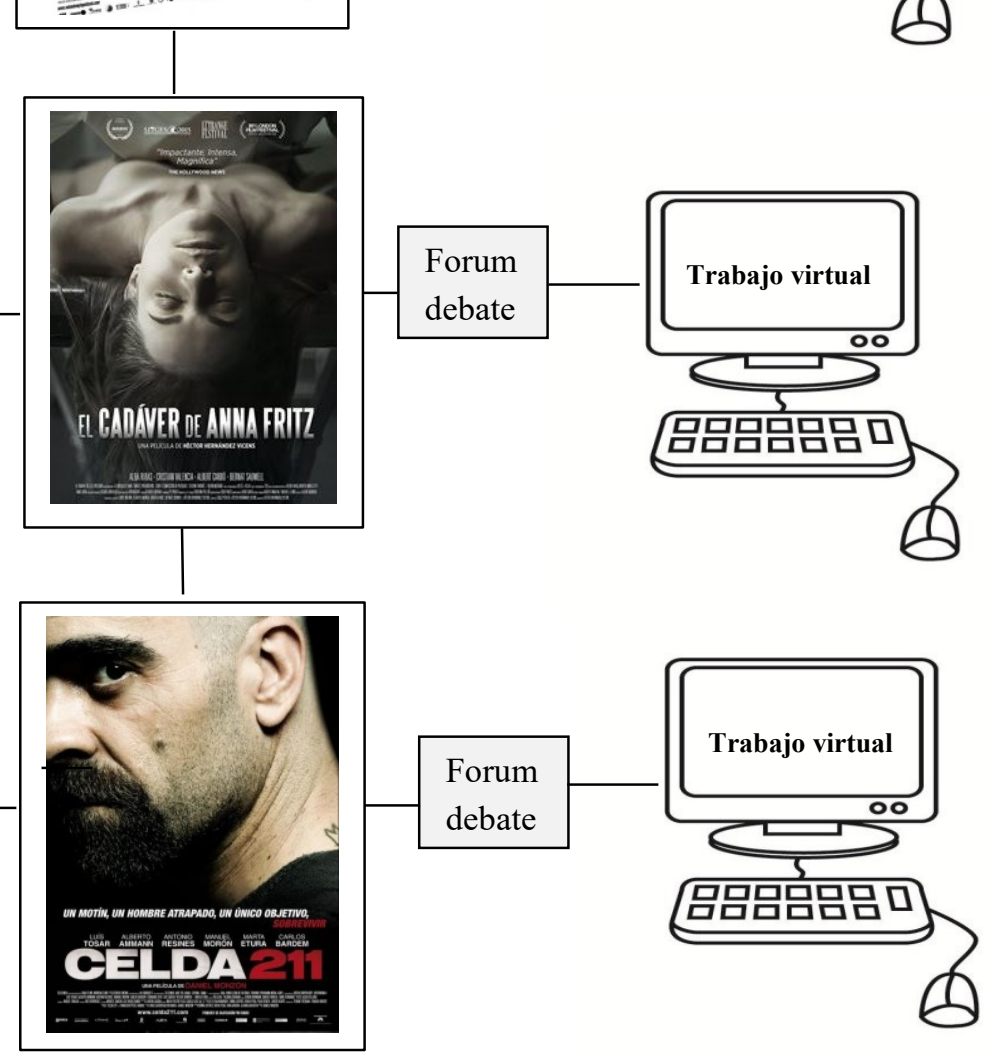

Fig. 1 Cronograma ejemplo del funcionamiento del Cineforum-virtual Criminologico en el primer trimestre del curso académico.

(c)) BY-NC-ND 2017, Universitat Politècnica de València 


\subsubsection{Procedimiento}

Antes de empezar el curso académico, el profesorado que imparte las asignaturas de $3^{\text {er }}$ curso del Grado de Criminología de la Universidad Europea de Valencia, formó una plataforma virtual y mantuvo una reunión para establecer el programa que se llevaría a cabo en el "Cineforum-virtual Criminológico", como actividad perteneciente a la parte práctica de las asignaturas, y así poder desarrollar además del aprendizaje de los conocimientos, las competencias prácticas.

Se elaboró un listado de peliculas, estudiando con exhaustividad los argumentos y sucesos que se desarrollan en las sinopsis de las mismas. Se encargó cada uno de los profesores/as, de visionar las películas susceptibles de introducir en el programa, para tener la seguridad de que eran adecuadas con los temas de las asignaturas. Una vez elaborado el listado, véase Tabla 1, se distribuyeron las proyecciones, los debates y los posteriores trabajos virtuales, a lo largo de los tres trimestres.

El primer día de clase, los profesores que forman parte de esta plataforma, explicaron a sus alumnos la actividad de "Cineforum-virtual Criminológico", su funcionamiento y un calendario de proyecciones y forum, fechas límites de utilización virtual de la película y de entregas de trabajos, teniendo en cuenta, que aunque se dispusiera de una película concreta hasta el último trimestre, por trabajar con ella en varias asignaturas, los trabajos o informes concretos de cada una de las mismas, sí que tenían fecha límite de entrega.

Las películas se proyectaron en aula a principio del último mes de cada trimestre (en algunos casos a mitad del trimestre), una vez que los estudiantes habían recibido los conomientos necesarios para poder analizar, valorar, y resolver, en su caso, lo que se le solicitó, según el argumento. Como indica el cronograma ejemplo de la Figura 1, el proceso fue el siguiente:

$1^{\circ}$. Explicación teórica de los contenidos.

$2^{\circ}$. Proyección de la película + fórum, con un guíon propuesto por cada profesor.

$3^{\circ}$. Trabajos individuales con posibilidad de visionar de nuevo la película en casa. En la plataforma, cada profesor indicó los trabajos a realizar según asignatura, mínimo tres. Ejemplos: valoración de posible enfermedad mental según la trayectoria de vida del protagonista, o del transcurso de los hechos; factores de riesgo o protección del delincuente; penas que deberían imponerse según un caso particular; qué victimología se desdibujaba desde el principio de los hechos o indicios; qué tipología de delitos se han visto y qué penas se derivarian, en su caso, con atenuantes o eximentes, o incluso posibilidad de inimputabilidad, etc.

$4^{\circ}$. Participación virtual una vez habían entregado los trabajos. Se abrió un foro de discusión sobre las problemáticas y las valoraciones realizadas por los alumnos, después de que los trabajos se presentaron en fecha. 
$5^{\circ}$. Sesión presencial sobre reflexiones y, en su caso, soluciones, de los trabajos solicitados.

\section{Resultados}

Para comprobar el aprendizaje y valoración de esta actividad como recurso formativo y complementario para el aprendizaje de las asignaturas de $3^{\circ}$ del Grado de Criminología, se pasó a los estudiantes un breve cuestionario de 5 ítems con valores cuantitativos de 0 a 10 . Se obtuvieron resultados muy satisfactorios:

Tabla 2. Cuestionario de valoración del “Cineforum-virtual Criminológico”

\begin{tabular}{lc}
\hline Items & Medias \\
\hline $\begin{array}{l}\text { 1. Con esta metodología he aprendido con más eficacia y relación } \\
\text { los contenidos de esta asignatura. }\end{array}$ & 9 \\
2. Me ha servido para venir a clase con más motivación. & 9 \\
$\begin{array}{l}\text { 3. Con esta metodología he puesto en práctica lo explicado en } \\
\text { clase de mejor forma que con casos escritos. }\end{array}$ & 10 \\
$\begin{array}{l}\text { 4. Me gustaría que el próximo curso se trabajara alguna asignatura } \\
\text { con esta metodología. }\end{array}$ & 10 \\
5. Recomendaría esta metodología. &
\end{tabular}

Por lo que se refiere a la evaluación de la actividad, se divide en dos partes. La entrega de los trabajos sobre la película, suponía un 30\% de la nota final de la asignatura correspondiente y otro $10 \%$ la participación en los debates y forum, tanto presenciales como virtuales. Un $10 \%$ fue para otras actividades y el $50 \%$ restante fue para el examen final escrito, con un formato de preguntas abiertas. Los resultados que se obtuvieron han sido muy satisfactorios, puesto que de los 11 alumnos, 9 han sacado la máxima clasificación sobre la actividad (4), 2 alumnos han sacado un 3. También se detectó que las notas de los exámenes escritos habían sido superiores que las del grupo del año anterior.

\section{Conclusiones}

La valoración global del profesorado al finalizar el curso, fue muy satisfactoria por varias razones. En primer lugar, por los resultados positivos de los exámenes finales y de los trabajos. En segundo lugar, por el carácter motivador que se respiraba en las aulas y en los debates; además de prácticar la capacidad de interrelacionar una problemática a varios 
ámbitos de trabajo, cuestión difícil de demostrar con otros recursos. Además, por la aplicación práctica de la legislación como solución a diversos acontecimientos y conflictos, así como para valorar las diferentes conductas criminales y los posibles estados psicológicos que pueden sufrir las personas para desarrollar ciertas acciones. La involucración de los alumnos ha sido muy llamativa, en comparación con otras actividades y el aprendizaje de gestión de las emociones para tomar decisiones, ha sido un experiencia para los alumnos muy práctica y necesaria para estos estudios.

Para finalizar, esta metodología, ha resultado positiva por la capacidad de conseguir con este recurso y el trabajo posterior, que los alumnos adquieran la mayoría de competencias que describe el Grado de Criminología.

\section{Referencias}

ALEXANDER, M., HALL, M. N., \& PETTICE, Y. J. (1993). Cinemeducation: an innovative approach to teaching psychosocial medical care. Family medicine, 26(7), 430-433.

BETANZO, P. B. (2010). "El Cine como herramienta eficaz para un aprendizaje concreto, activo y reflexivo: una experiencia en Aula“. Buenos Aires: Congreso Iberoamericano de Educación.

BOSCH, F., \& BAÑOS, J. E. (1999). Empleo de películas comerciales en la docencia de la farmacología. Educ Med, 4(2), 197.

DE LA TORRE, S. (1996). Cine formativo. Una estrategia innovadora para los docentes. Editorial Octaedro, España.

DOMÍNGUEZ, M. D. C. P., \& GUERCIA, C. U. (2005). Cine y juventud: una propuesta educativa integral. Revista de estudios de juventud, (68), 73-90.

FERNANDEZ PALOMARES, F. Y OLMEDO REINOSO, A. (2003). "Barbiana y las «escuelas japonesas: apoyos audiovisuales para ayudar a comprender la dimensión social de la enseñanza“, $X$ Conferencia de Sociología de la Educación, Valencia, 18-20 septiembre.

GARCÍA-SÁNCHEZ, J. E., FRESNADILLO, M. J., \& GARCÍA-SÁNCHEZ, E. (2002). El cine en la docencia de las enfermedades infecciosas y la microbiología clínica. Enfermedades infecciosas y Microbiología clínica, 20(8), 403-406.

GONZÁLEZ, E. M. Á. (2015). El cine como metodología docente aplicable a la enseñanza del Derecho Administrativo. REJIE: Revista Jurídica de Investigación e Innovación Educativa, (11), 97 109.

ICART-ISERN, M. T. (2008). "Metodología de la investigación y cine comercial: claves de una experiencia docente". Educación médica, 11(1), 13-18.

MARTÍNEZ ÁlVAREZ, J. (2003). Peliculas para usar en el aula. Madrid: UNED

RODRÍGUEZ, R. (2006). Diseño de entornos para el desarrollo de la autonomía en el aprendizaje. Aula Abierta, 87, 89-104

SALAS, D. P., \& CASAREJOS, M. N. G. (2013). El cine como herramienta de aprendizaje en el aula. Claves de una experiencia docente multidisciplinar en el ámbito económico. Revista Internacional de Organizaciones, (10), 181-197.

\section{Películas}

(cc)) EY-NG-ND 2017, Universitat Politècnica de València 
Cineforum-virtual criminológico como estrategía de aprendizaje teorico-práctico para el análisis del comportamiento criminal en el Grado de Criminología

Volando Voy (Dir. Miguel Albaladejo). Mediapro. 2006.

El cadáver de Anna Fritz (Dir. Héctor Hernández). A Contraluz Films / Benecé Produccions / Corte y Confección de Películas / Playtime Movies / Silendum Films. 2015.

Celda 211 (Dir. Daniel Monzón). Coproducción España-Francia; La Fabrique de Films / Morena Films / Telecinco Cinema / Vaca Films. 2009.

(c) EY-NC-ND 2017, Universitat Politècnica de València 